\title{
SIMULATION OF MOVEMENT OF THE DEVICE WITH PASSIVE VIBRATION ISOLATION
}

\author{
IGOR N. SHARDAKOV ${ }^{1}$, IRINA O. GLOT ${ }^{2}$ AND ALEKSEY P. SHESTAKOV ${ }^{3}$
}

\author{
1, Korolev str. Perm, Russia, 614018 \\ shardakov@icmm.ru \\ ${ }^{2}$ Institute of Continuous Media Mechanics of the Ural Branch of Russian Academy of Science \\ 1, Korolev str. Perm, Russia, 614018 \\ glot@icmm.ru \\ ${ }^{3}$ Institute of Continuous Media Mechanics of the Ural Branch of Russian Academy of Science \\ 1, Korolev str. Perm, Russia, 614018 \\ shap@icmm.ru
}

Key words: Passive Vibration Isolation, Angular Vibrations, Dampers, Mathematical Model.

\begin{abstract}
Modern electronic systems, computer hardware and navigation equipment on board moving objects can be subjected to significant mechanical impulse and vibrational impacts. These impacts can introduce additional errors in readings of devices, and sometimes lead to their mechanical failure. One of the effective ways to solve the problem is to apply the method of passive vibration protection, which makes it possible to reduce vibrations due to the use of damping elements. This paper examines the vibration response of a device mounted on a moving platform. The device is protected against vibration by 4 dampers. The platform is subjected to translational motions in three mutually orthogonal directions. This leads to the appearance of coupled translational and rotational vibrations of the protected unit. The problem is solved within the framework of the general theory of the dynamic of a rigid body. The paper presents the results of numerical experiments, in which the intensity of rotational vibrations of the protected unit is investigated depending on various mechanical characteristics of the system. Admissible variation of these characteristics, at which the angular acceleration of the protected unit remains below a limit value, has been determined.
\end{abstract}

\section{INTRODUCTION}

Multi-purpose electronic devices, such as control units, navigation equipment, measuring units, etc., have found wide application in different types of modern vehicles. During their operation they often experience considerable mechanical stresses, including mechanical impulses, vibrations, shocks, linear overloads, acoustic noise. These impacts significantly impair the characteristics of high-precision apparatus, introduce additional errors in the readings, and sometimes can lead to mechanical failure of the devices [1, 2]. One of the common ways to solve the problem is to apply the passive vibration protection technique, 
which makes it possible to reduce vibrations of the protected unit through the use of damping elements. Devices requiring vibration protection are often combined into a single unit, which is mounted on a supporting structure using a damping system. An appropriate selection of the damper characteristics can significantly reduce the intensity of the impact on the protected module. Under certain external actions on the supporting structure the coupled vibration modes, involving both the translational and rotational vibrations, can also arise in the protected unit. Such modes are dangerous because mechanical energy is transferred from one vibration mode to others, causing a sharp increase in the vibration intensity. For many electronic devices, angular vibrations are considered highly undesirable, and designers impose restrictions just on the angular kinematic characteristics (namely, displacements, angles of rotation and the corresponding speeds and accelerations) $[3,4]$.

The unit protected against vibrations can be considered as a rigid body with 6 degrees of freedom: 3 translational and 3 rotational. In organizing an effective vibration protection, there are several important points to consider. The properties of the damping materials must be appropriately selected to ensure the most efficient energy dissipation. It is necessary to locate dampers in such a way that the mutual influence of rotational and translational vibration modes is minimal. The solution to these problems can be obtained by mathematical modeling of the dynamic response of the protected module to typical external actions and subsequent analysis of the obtained results.

This paper examines the movement of a block of electronic devices combined into a single module mounted on

dampers, which separa translational vibrations translational and rotational dissipative properties. By changing these prop dampers, one can keep the vibrations of the proten on the operating conditions of the protected unit, the value of its angular acceleration is

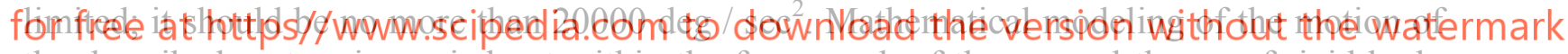
the described system is carried out within the framework of the general theory of rigid body dynamics.

\section{MATHEMATICAL MODEL}

A schematic block diagram of the dynamic interaction of the protected block with a basic structure is shown in Figure 1a. Protected unit 1 is attached to the rigid platform 2, which is mounted on the supporting structure. The unit is connected to the platform through four dampers A, B, C, D. The platform can move in three mutually perpendicular directions. The stiffness of the dampers is considerably less than the stiffness of the block and platform, and therefore the protected block can be treated as an absolutely rigid body with mass $m$ and the corresponding moments of inertia. It is assumed that the dumpers are weightless and have elastic and dissipative properties. The computational model of the device is given in Figure 1b. Within the framework of this model, the protected unit (solid block) is represented as a point mass 1 with a specified direction of the main axes and the values of the main moments of inertia. The point mass is connected to the dampers by weightless rigid rods 2 , which are directed along the axis lines of the dampers. The dampers are attached to a rigid 
weightless platform 4 using weightless rigid rods 3 . The dampers are considered as weightless hinges with 6 degrees of freedom ( 3 translational and 3 rotational). The elastic and dissipative properties of the dampers are set by two corresponding coefficients for each degree of freedom.
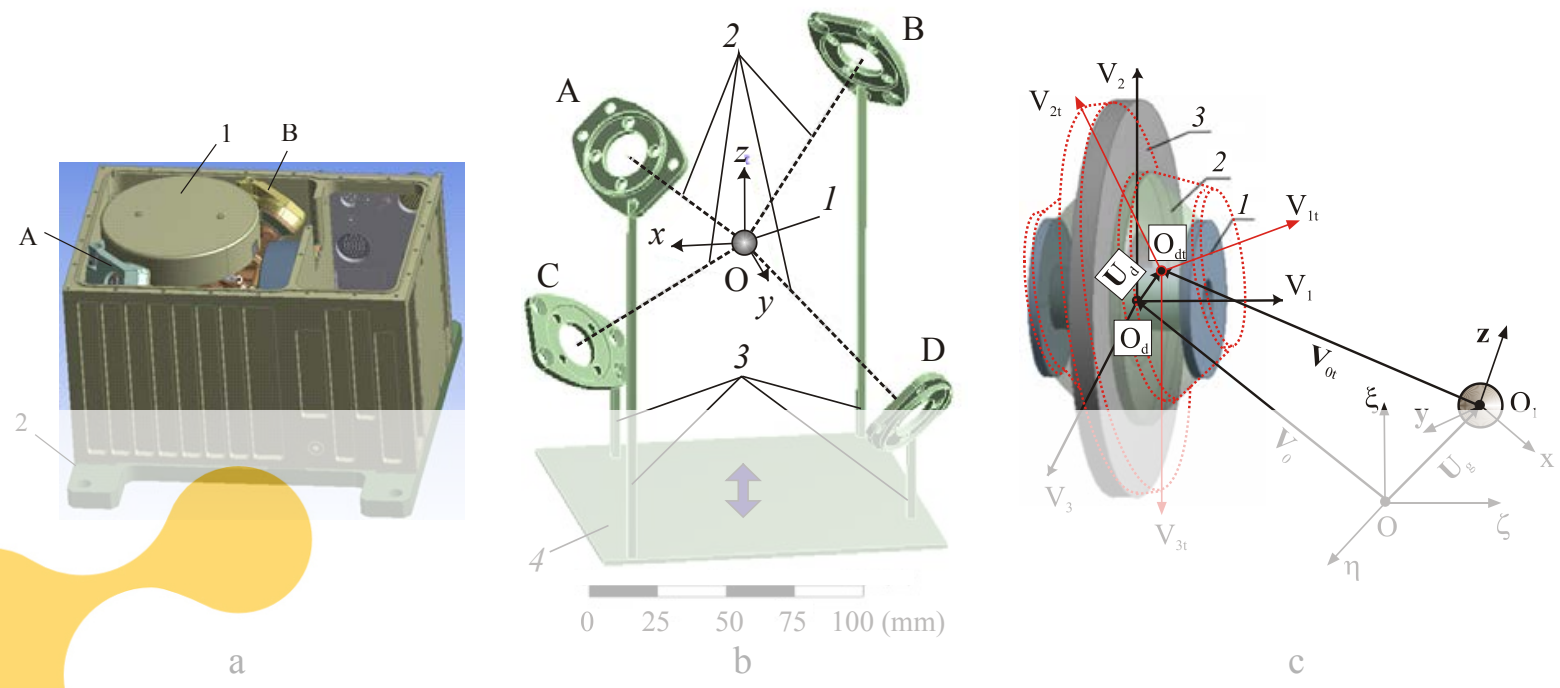

Figure 1: Vibration-protected unit fixed on the supporting structure (a), computational model (b) and damper
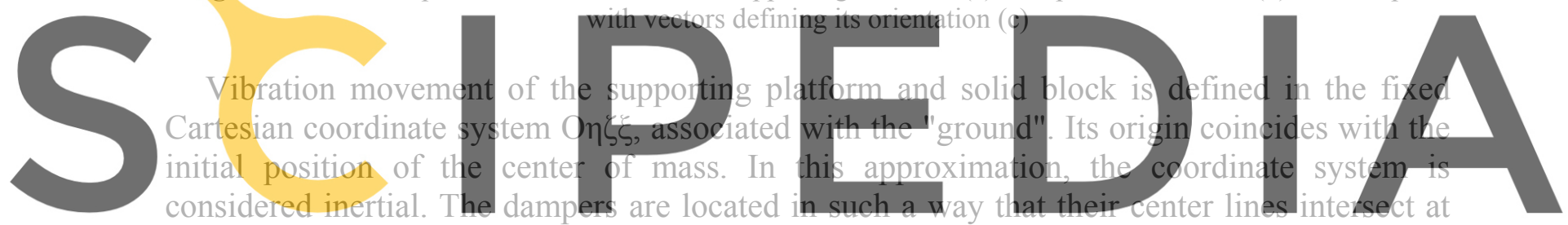

one point and this point is called the center of stiffness of the system. At the initial moment of

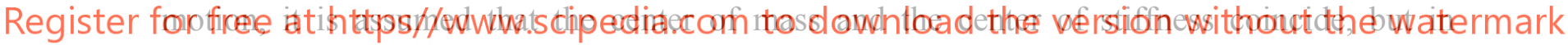
general, they may not coincide.

The damper design is shown in Figure 1c. The damper is attached on one side to the protected block using a rigid axis 1 , and on the other side to the supporting structure using a rigid washer 3. Deformable element 2, which has elastic and dissipative properties, provides vibration damping. When the system moves, the point mass is displaced relative to the fixed coordinate system and the rigid elements 1 and 3 are displaced relative to each other. The relative position of the damper elements at an arbitrary moment is shown in Figure 1c with a red dotted line. The movement of the damper elements relative to the point mass can be conveniently described in a moving coordinate system $\mathrm{O}_{1} \mathrm{xyz}$, the origin of which is associated with the point mass. At the initial moment, the moving coordinate system coincides with the fixed one, but then it can perform translational and rotational motions relative to the fixed coordinate system.

A mathematical model of motion of the presented system is formulated within the framework of the classical theory of rigid body dynamics [5]. The translational motion of the protected block with respect to the fixed coordinate system $O \eta \zeta \xi$ is described by the following equations: 


$$
m \frac{\partial^{2} U_{\xi}}{\partial t^{2}}=F_{\xi}, \quad m \frac{\partial^{2} U_{\eta}}{\partial t^{2}}=F_{\eta}, \quad m \frac{\partial^{2} U_{\zeta}}{\partial t^{2}}=F_{\zeta}
$$

where $t$ is time, $m$ is the solid block mass; $U_{\xi}, U_{\eta}, U_{\zeta}$ are the components of the vector of displacement of the point mass, and $F_{\xi}, F_{\eta}, F_{\zeta}$ are the components of external forces.

The rotational motion of the protected block is described in a moving coordinate system $\mathrm{O}_{1} \mathrm{xyz}$ by the following equations:

$$
I_{x} \frac{\partial \omega_{x}}{\partial t}+\left(I_{z}-I_{y}\right) \omega_{y} \omega_{z}=M_{x}, \quad I_{y} \frac{\partial \omega_{y}}{\partial t}+\left(I_{x}-I_{z}\right) \omega_{x} \omega_{z}=M_{y}, \quad I_{z} \frac{\partial \omega_{z}}{\partial t}+\left(I_{y}-I_{x}\right) \omega_{x} \omega_{y}=M_{z},
$$

where $I_{x}, I_{y}, I_{z}$ and $\omega_{x}, \omega_{y}, \omega_{z}$ are the main moments of inertia and the angular velocities of the solid block, and $M_{x}, M_{y}, M_{z}$ are the moments applied to the block.

The position of the moving coordinate system relative to the fixed one is determined using Tait-Bryan angles $\psi, \theta, \varphi$, which are related to angular velocities as follows:

$$
\frac{\partial \psi}{\partial t}=\frac{\cos (\varphi)}{\cos (\theta)} \omega_{y}-\frac{\sin (\varphi)}{\cos (\theta)} \omega_{z}, \quad \frac{\partial \theta}{\partial t}=\sin (\varphi) \omega_{y}+\cos (\varphi) \omega_{z}, \quad \frac{\partial \varphi}{\partial t}=\omega_{x}-\cos (\varphi) \operatorname{tg}(\theta) \omega_{y}+\sin (\varphi) \operatorname{tg}(\theta) \omega_{z} .
$$

\section{Initial conditions are set as follows:}

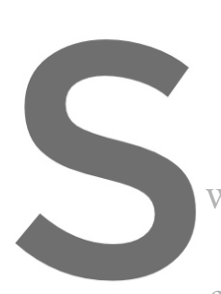
$\left.\omega_{x}\right|_{t=0}=\omega_{x}^{0}$
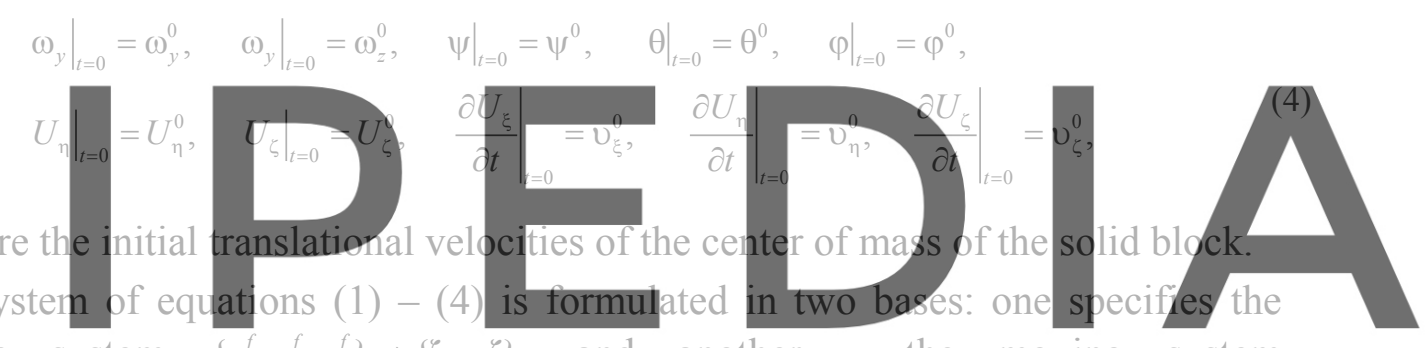

fixed coordinate system

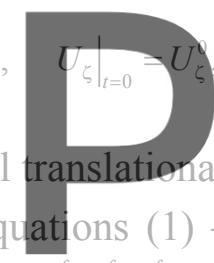

and

another

the moving system

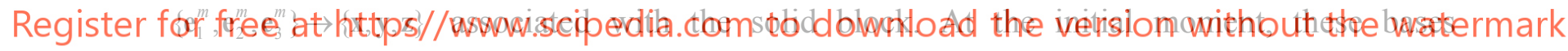

coincide with each other. Hereinafter, the superscripts $f$ and $m$ denote belonging to the fixed

and moving basis, respectively. The direct and inverse transformation of the bases is carried out using the rotation matrix $\mathbf{R}$

$$
\mathbf{R}=\left[\begin{array}{ccc}
\cos (\psi) \cos (\theta) & \sin (\varphi) \sin (\psi)-\cos (\varphi) \cos (\psi) \sin (\theta) & \cos (\varphi) \sin (\psi)+\sin (\varphi) \cos (\psi) \sin (\theta) \\
\sin (\theta) & \cos (\theta) \cos (\varphi) & -\cos (\theta) \sin (\varphi) \\
-\sin (\psi) \cos (\theta) & \sin (\varphi) \cos (\psi)+\cos (\varphi) \sin (\psi) \sin (\theta) & \cos (\varphi) \cos (\psi)-\sin (\varphi) \sin (\psi) \sin (\theta)
\end{array}\right]
$$

An arbitrary vector $\mathrm{V}$ defined in the moving basis can be written in the fixed basis: $\mathbf{V}^{f}=\mathbf{R} \cdot \mathbf{V}^{m}$. The reverse vector transformation is represented as $\mathbf{V}^{m}=\mathbf{R}^{T} \cdot \mathbf{V}^{f}$ (where ${ }^{T}$ denotes a transpose operation).

At the initial moment of time the orientation of the damper is determined by a set of unit vectors $\mathbf{V}_{1}, \mathbf{V}_{2}, \mathbf{V}_{3}$ rigidly connected to the damper axis 1 (Figure 1c). The position of the damper relative to the point mass is determined by the radius vector $\mathbf{V}_{0}$ directed from the origin of the moving coordinate system $\mathrm{O}_{1}$ (wich coinsides with $\mathrm{O}$ at the initial moment) to the damper center $\mathrm{O}_{d}$. At an arbitrary moment, the new position of the point mass is $\mathrm{O}_{1 t}$ and 
the new position of the damper center is $\mathrm{O}_{d t}$. At the same time the new damper orientation is determined by the vectors $\mathbf{V}_{1 t}, \mathbf{V}_{2 t}, \mathbf{V}_{3 t}$ (the triplet of red arrows in Figure 1c). The new position of the damper center relative to the point mass is determined by the vector $\mathbf{V}_{0 t}$.

The triplet of vectors $\mathbf{V}_{1}, \mathbf{V}_{2}, \mathbf{V}_{3}$ connected with the damper axis 1 moves with it. At an arbitrary moment, the system of these vectors and the vector $\mathbf{V}_{0}$ in the fixed coordinate system are defined as follows:

$$
\left[\mathbf{V}_{1 t}^{f}, \mathbf{V}_{2 t}^{f}, \mathbf{V}_{3 t}^{f}\right]=\mathbf{R} \cdot\left[\mathbf{V}_{1}^{f}, \mathbf{V}_{2}^{f}, \mathbf{V}_{3}^{f}\right], \quad \mathbf{V}_{0 t}^{f}=\mathbf{R} \cdot \mathbf{V}_{0}^{f}+\mathbf{U}_{g}^{f},
$$

where $\mathbf{U}_{g}^{f}$ is the vector of displacement of the point mass.

The displacement of the geometric center of the damper is calculated by the formula

$$
\mathbf{U}_{d}=\mathbf{V}_{0 t}-\mathbf{V}_{0}+\mathbf{U}_{g}
$$

The angular and translational velocities of the rigid part of the damper relative to its initial position are calculated by the formulas:

$$
\boldsymbol{\omega}_{d}^{m}=\left[\begin{array}{lll}
\mathbf{V}_{1}^{f} & \mathbf{V}_{2}^{f} & \mathbf{V}_{3}^{f}
\end{array}\right]^{T} \cdot \boldsymbol{\omega}_{g}^{m}, \quad \mathbf{v}_{d}^{f}=\mathbf{v}_{g}^{f}+\boldsymbol{\omega}_{g}^{f} \times \mathbf{V}_{0}^{f},
$$

Where $\omega_{d}^{m}$ and $\omega_{g}^{m}$ are the angular velocities of the damper and the point mass relative to the

moving coordinate system, $v_{d}^{f}$ is the translational velocity of the damper and $\omega$ angular velocity of the point mass relative to the fixed coordinate system.
The reactions arising in the damper are calculated as follows:

- the force and momentum caused by the

$\mathbb{F}_{\mu}^{f}=C_{1 u}\left(\mathbb{U}_{d}^{f} \cdot \mathbf{V}_{1}^{f}\right) \mathbf{V}_{1}^{f}+C_{2 u}\left(\mathbb{U}_{d}^{f} \cdot \mathbf{V}_{2}^{f}\right) \mathbf{V}_{2}^{f}+C_{3 u}\left(\mathbb{U}_{d}^{f} \cdot \mathbf{V}_{3}^{f}\right) \mathbf{V}_{3}^{f}, \quad \mathbf{M}_{u}^{m}=\mathbf{V}_{0}^{f} \times \mathbb{F}_{u}^{m}$,

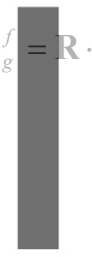

$\omega_{g}^{m}$ is

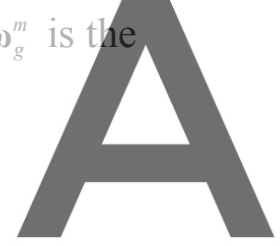

(5)

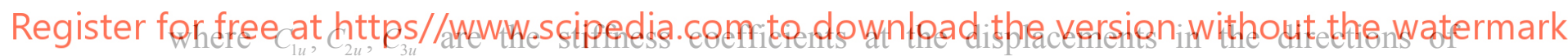

$\mathbf{V}_{1}^{f}, \mathbf{V}_{2}^{f}, \mathbf{V}_{3}^{f}$ axes; $\mathbb{F}_{u}^{m}=\mathbb{R}^{T} \cdot \mathbb{F}_{u}^{f}$ is the force vector in the moving coordinate system;

- the force and momentum caused by the damper rotation:

$\mathbf{F}_{\mathrm{v}}^{f}=K_{1 \mathrm{v}}\left(\mathbf{v}_{d}^{f} \cdot \mathbf{V}_{1}^{f}\right) \mathbf{V}_{1}^{f}+K_{2 \mathrm{v}}\left(\mathbf{v}_{d}^{f} \cdot \mathbf{V}_{2}^{f}\right) \mathbf{V}_{2}^{f}+K_{3 \mathrm{v}}\left(\mathbf{v}_{d}^{f} \cdot \mathbf{V}_{3}^{f}\right) \mathbf{V}_{3}^{f}, \quad \mathbf{M}_{a}^{m}=C_{1 a} A_{1} \mathbf{V}_{1}^{f}+C_{1 a} A_{2} \mathbf{V}_{2}^{f}+C_{1 a} A_{3} \mathbf{V}_{3}^{f}$,

where $K_{1 v}, K_{2 v}, K_{3 v}$ are the viscous friction coefficients at the displacements in the directions of the $\mathbf{V}_{1}^{f}, \mathbf{V}_{2}^{f}, \mathbf{V}_{3}^{f}$ axes; $C_{1 a}, C_{2 a}, C_{3 a}$ are the stiffness coefficients in the case when rotation is executed about the axes $\mathbf{V}_{1}^{f}, \mathbf{V}_{2}^{f}, \mathbf{V}_{3}^{f}$;

- the momentum caused by the force reaction to the damper displacement and rotation in a viscous medium:

$$
\mathbf{M}_{\mathrm{v}}^{m}=\mathbf{V}_{0}^{f} \times\left(\mathbf{R}^{T} \cdot \mathbf{F}_{\mathrm{v}}^{f}\right), \quad \mathbf{M}_{\mathrm{v} a}^{m}=\left\{K_{1 a} \cdot \omega_{1 d}^{m}, K_{2 a} \cdot \omega_{2 d}^{m}, K_{3 a} \cdot \omega_{3 d}^{m}\right\}^{T},
$$

where $K_{10}, K_{2 v}, K_{3 v}$ are the viscous friction coefficients, when rotation occurs in the directions of the $\mathbf{V}_{1}^{f}, \mathbf{V}_{2}^{f}, \mathbf{V}_{3}^{f}$ axes. 
For an arbitrary damper, the resulting vector of the reaction force, reduced to the center of mass of the rigid body, as well as the resulting momentum, are written as follows:

$$
\mathbf{F}^{f}=\mathbf{F}_{u}^{f}+\mathbf{F}_{\mathrm{v}}^{f}, \quad \mathbf{M}^{m}=\mathbf{M}_{u}^{m}+\mathbf{M}_{a}^{m}+\mathbf{M}_{\mathrm{v}}^{m}+\mathbf{M}_{\mathrm{va}}^{m} .
$$

The total force and the total momentum acting on the system are equal to the sum of the reactions of $N$ dampers:

$$
\mathbf{F}_{\text {sum }}^{f}=\sum_{i=1}^{N} \mathbf{F}_{i}^{f}, \quad \mathbf{M}_{\text {sum }}^{m}=\sum_{i=1}^{N} \mathbf{M}_{i}^{m} .
$$

The total reactions $\left\{\begin{array}{llll}F_{\xi} & F_{\eta} & F_{\zeta}\end{array}\right\}^{T}=\mathbf{F}_{\text {sum }}^{f}$ and $\left\{\begin{array}{lll}M_{x} & M_{y} & M_{z}\end{array}\right\}^{T}=\mathbf{M}_{\text {sum }}^{m}$ are substituted into equations (1) and (2). The resulting system of differential equations (1) - (3) with initial conditions (4) was solved numerically by the Runge-Kutta method of the 4th order using the MATLAB software.

\section{SIMULATION RESULTS}

Based on the developed mathematical model, a series of numerical experiments was performed to investigate the motion of the protected block caused by vibrations of the supporting platform along the vertical axis. The frequency range of the vibrations was $150-350 \mathrm{~Hz}$, the acceleration amplitude was $3 \mathrm{~g}$. In the following discussion, the $\mathrm{z}-$ axis is directed vertically upward, the $x$ - and $y$ - axes are in the horizontal plane.

The configuration of block coincides with the the same, is ideal (or basi numerical experiment protected block relative
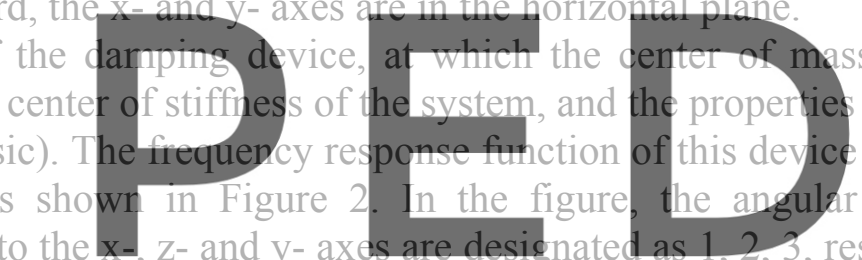

seen from the figure, in this case the maximum amplitudes of angular velocities are 0.025 ,

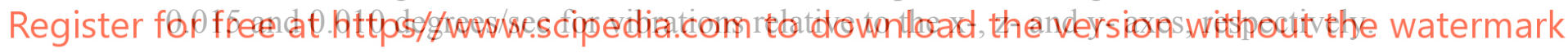

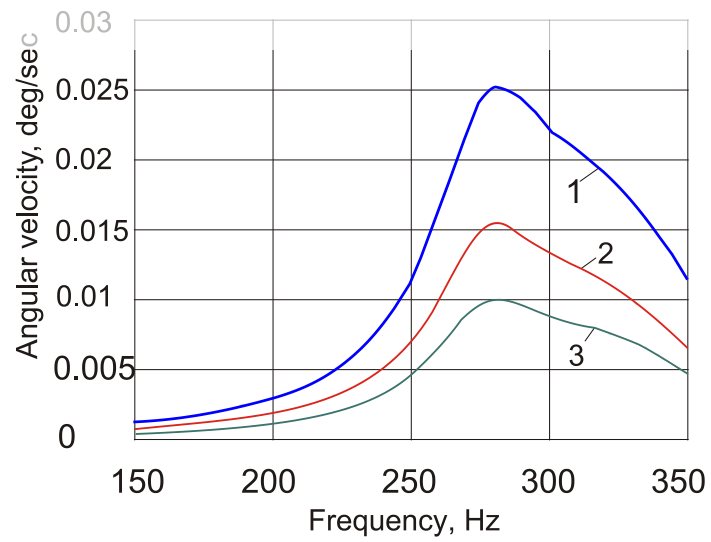

Figure 2: Frequency response of angular velocities for the basic design of the damping device:

$$
1-\omega_{x}, 2-\omega_{z}, 3-\omega_{y}
$$


The configuration of real devices may differ from the basic one. The mechanical characteristics of the real device of similar design were measured in the earlier experiment [6]. The relative position of the center of mass and the center of stiffness of the device was measured, as well as the stiffness and dissipation characteristics of dampers. Further we discuss such characteristics as axial and radial stiffness coefficient $\left(C_{a}\right.$ and $C_{r}$ ). These values correspond to the coefficients $C_{1 u}$ and $C_{2 u}=C_{3 u}$ from equation (5), which characterize the stiffness of the damper when its elements are displaced in the direction of the damper axis $\mathbf{V}_{1}$ and radii $\mathbf{V}_{2}$ and $\mathbf{V}_{3}$. It was found that the center of mass of the protected block is displaced relative to the center of stiffness by $\Delta \mathrm{M}=2.6 \mathrm{~mm}(-1.5,-1.4$ and $-1.6 \mathrm{~mm}$ in the direction of the $x-, y-$ and $z-$ axes, respectively). The values of the stiffness coefficient in the axial and radial directions for the 4 dampers used in the experimental sample are different. The average values of these characteristics and their standard deviations are given in Table 1.

\begin{tabular}{|ccc} 
& Table 1: Experimental values of damper characteristics \\
\hline & Axial stiffness coefficient, $C_{a}$ & Radial stiffness coefficient $C_{r}$ \\
\hline $\begin{array}{l}\text { Mean value, } \mathrm{N} / \mathrm{m} \\
\text { Standard deviation } \\
(\% \text { of the Mean) }\end{array}$ & $2,87 \cdot 10^{6}$ & $2,16 \cdot 10^{6}$ \\
\hline
\end{tabular}

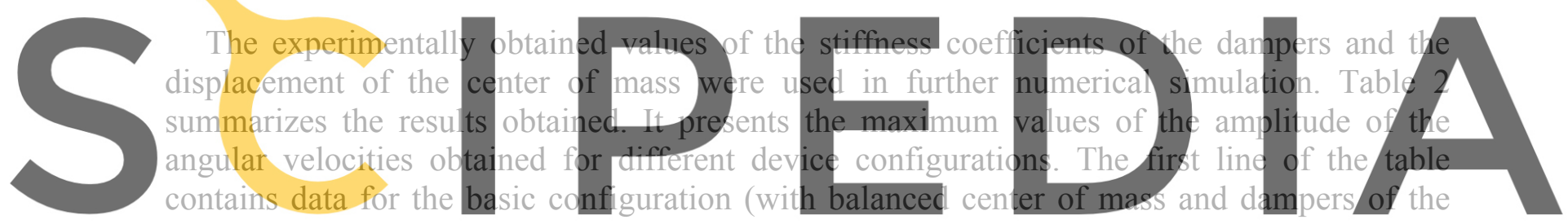
same stiffness $C_{a}=2,87 \cdot 10^{6} \mathrm{~N} / \mathrm{m}$ and $\left.C_{r}=2,16 \cdot 10^{6} \mathrm{~N} / \mathrm{m}\right)$. The second line corresponds to the

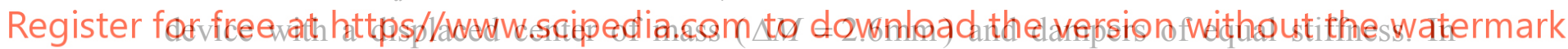
addition, consideration was given to the cases when the center of mass was displeased and the stiffness coefficients of one damper were increased or decreased by the value of standard deviation $(8.07 \%$ for the axial stiffness coefficient and $6.48 \%$ for the radial stiffness coefficient). Literal designation of the dampers with the changed stiffness values is given in the left column of the table; "+" means an increased value of the coefficients, and "-" a reduced value.

It can be seen from the table that the unbalanced centers of mass and stiffness of the system leads to a $200 \mathrm{x}$ increase in the angular velocity of the protected unit compared to the basic configuration. The variation in the properties of the dampers by the standard deviation value can additionally increase the angular velocity by a factor of about 5 .

Of particular interest is studying how the angular accelerations of the protected unit change due to variations introduced in the mechanical characteristics of the system (the ratio of the stiffness coefficients of the dampers and the mutual location of the centers of mass and stiffness). It is just the angular acceleration that is the limiting parameter specifying the design tolerances. When the device is in operation, its value should not exceed $20000 \mathrm{deg} / \mathrm{sec}^{2}$. 
Table 2: Angular velocity of the solid block for different values of damper stiffness

\begin{tabular}{cccc}
\hline \multirow{2}{*}{$\begin{array}{c}\text { Variable stiffness } \\
\text { damper }\end{array}$} & \multicolumn{3}{c}{ Maximum amplitude of angular velocity, deg/sec } \\
\cline { 2 - 4 } & $V_{x}$ & $V_{y}$ & $V_{z}$ \\
\hline Basic design & 0.025 & 0.010 & 0.015 \\
\hline No & 5.2 & 5.6 & 0.2 \\
\hline A- & 1.8 & 2.1 & 0.2 \\
\hline B- & 9.0 & 9.4 & 0.3 \\
\hline C- & 5.5 & 10.0 & 4.7 \\
\hline D- & 9.2 & 5.2 & 4.1 \\
\hline A+ & 7.0 & 7.5 & 0.2 \\
\hline B + & 3.7 & 3.4 & 0.3 \\
\hline C + & 7.7 & 5.0 & 2.6 \\
\hline D+ & 8.1 & 4.9 & 3.0
\end{tabular}

Modeling of the response of the protected module to platform vibrations in the vertical direction has shown that the system exhibits the least sensitivity to the displacement of the center of mass in the direction coinciding with the direction of external vibrations A displacement of the center of mass in the horizontal plane ( $\mathrm{x}$ and $\mathrm{y}$ directions) by $0.5 \mathrm{~mm}$ causes vibrations of the protected unit with an angular acceleration of $4700 \mathrm{deg} / \mathrm{sec}^{2}$, and similar displacements along the vertical axis $125 \mathrm{deg} / \mathrm{sec}^{2}$, i.e. 37 times less. Figure 3 a shows how the maximum ang $\mathrm{x}$ - axis. In this case, achieved when the center of missien

variations in the angula one damper. In this ca
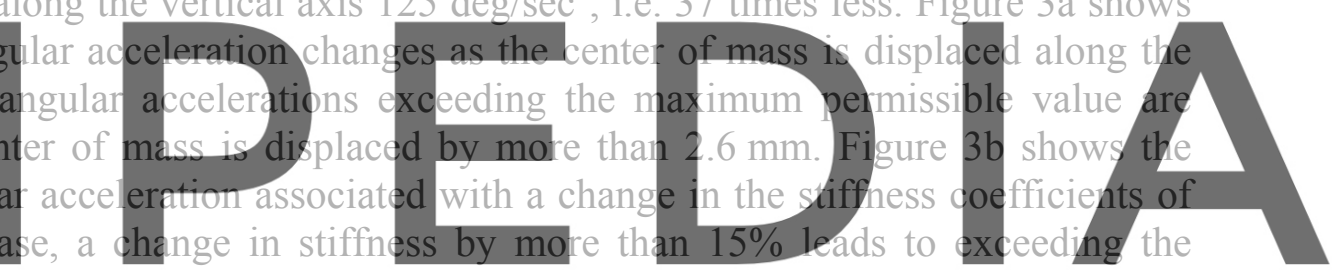

permissible value of the angular acceleration.

Register for free at https//www.scipedia.com to downgloged the version without the watermark

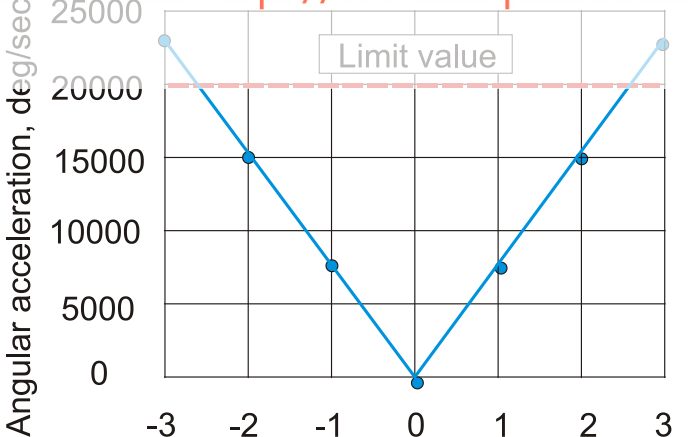

Center of mass displacement $\Delta \mathrm{x}, \mathrm{mm}$

a

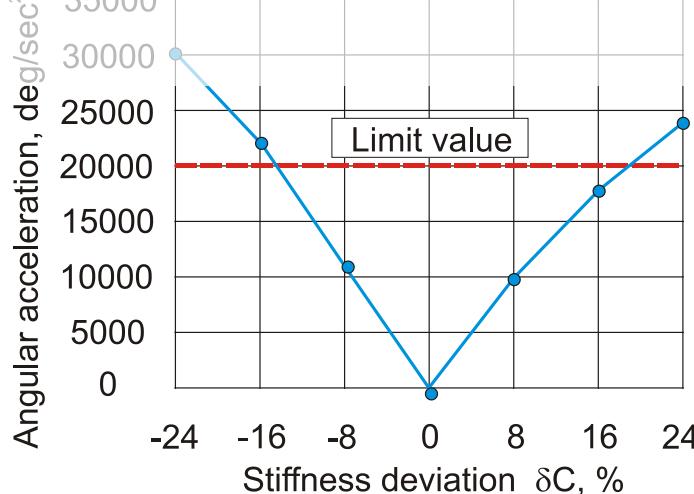

$\mathrm{b}$

Figure 3: Change in the maximum angular accelerations associated with the displacement of the center of mass along the $\mathrm{x}$-axis (a) and with a change in the stiffness coefficient of one damper (b)

An important factor determining the motion of the protected block is the interrelation of rotational and translational vibration modes. Numerical simulation allows us to gain a more 
clear insight into this phenomenon. A device with a center of mass displaced by $2 \mathrm{~mm}$ along the $\mathrm{x}$-axis relative to the balanced state was subjected to external vibrations along the vertical axis with acceleration amplitude of $3 \mathrm{~g}$. In this case, the protected block performed motion with a translational component in the $\mathrm{z}$ direction and angular vibrations, the most intense of which were the vibrations relative to the $y$ - axis. Figure 4 shows the calculated frequency response functions corresponding to the translational along the z-axis (a) and rotational relative to the y- axis (b) vibration modes. The curves in the figures correspond to different values of the coefficient $\mathrm{K}$ which means how much the axial stiffness coefficient of the dampers differs from the base value. In numerical experiment, we used $\mathrm{K}=0,0.5$, 1 , and 1.5 . The value $\mathrm{K}=1$ corresponds to the average values of stiffness given in Table 1 $\left(C_{a}=2,87 \cdot 10^{6} \mathrm{~N} / \mathrm{m}\right.$ and $\left.C_{r}=2,16 \cdot 10^{6} \mathrm{~N} / \mathrm{m}\right)$.
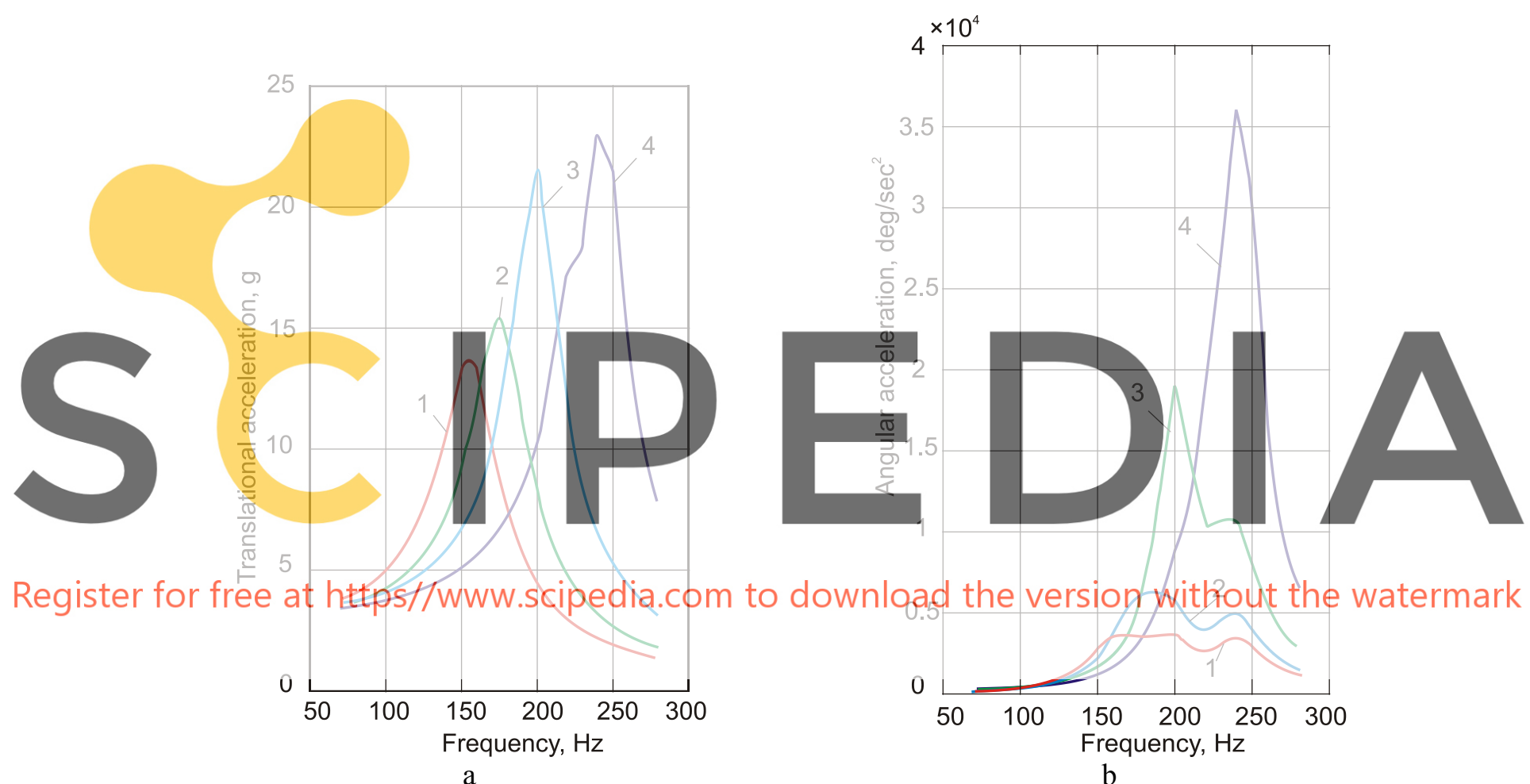

Register for free at https /www.scipedia.com to download the version without the watermark

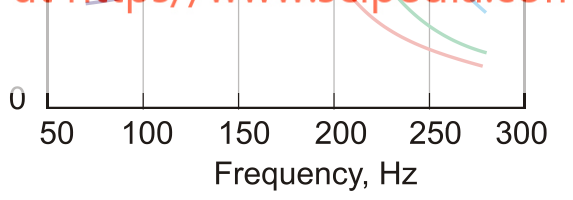

a

b

Figure 4: Frequency response of accelerations for translational (a) and rotational (b) vibrations at different values of $K$ : 0 (1), 0.5 (2), $1.0(3), 1.5$ (4)

Numerical modeling shows that the intensity of translational vibrations of the protected block is largely determined by the axial stiffness of the dampers. By changing the stiffness from 0 to the value, which is 1.5 times higher than the "base" value, one can change the linear acceleration from $13 \mathrm{~g}$ to $23 \mathrm{~g}$ (Figure $4 \mathrm{a}$ ). In this case, the resonant frequency varies from 150 to $240 \mathrm{~Hz}$. An increase in the stiffness of the dampers leads to a significant increase in the intensity of rotational vibrations, but the corresponding resonant frequency does not change substantially (Figure 4b). Thus, as the stiffness of the dampers increases, the resonant frequencies corresponding to the translational and rotational modes converge. At the same time, there is a significant increase in angular accelerations. With a 1.5 -fold increase in the 
stiffness of the dampers, the resonant frequencies of the translational and rotational vibrations practically coincide, and the amplitude of the angular accelerations at the resonant frequency is $\sim 10$ times higher than the value observed for a structure with dampers of zero stiffness.

The nature of vibrations of a mechanical system incorporating dampers significantly depends on the material of the damping element (Figure 1c, element 2). The damping element is made of a viscoelastic material, whose properties are characterized by the modulus of elasticity $\mathrm{E}$ and the dissipation factor (mechanical loss tangent LT). Numerical simulation allowed us to analyze the influence of each of these factors on the dynamics of the protected module. We analyzed the movements of a device, the center of mass of which is displaced from the center of stiffness by $5 \mathrm{~mm}$ and 4 dampers have the same characteristics. An external excitation acceleration with an amplitude of $3 \mathrm{~g}$ was applied in the vertical direction. The graph in Figure 5a shows the dependence of the maximum angular acceleration of the protected block on the loss tangent. The angular acceleration decreases significantly with increasing LT. An increase in LT by a factor of 10 (from 0.1 to 1 ) is accompanied by a decrease in the angular acceleration by more than 100 times. On the other hand, the dependence of rotational vibrations on the elastic modulus is insignificant. Figures $5 \mathrm{~b}$ and $5 \mathrm{c}$ show the dependences obtained at two values of loss tangent ( 0.1 and 1$)$. In both cases, an increase in the elastic modulus by a factor of 40 is accompanied by a change in the angular acceleration by no more than $15 \%$. Thus, the numerical experiment showed that the main factor affecting the magnitude of the angular acceleration is the dissipative characteristic of

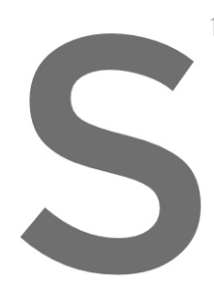
the damping material

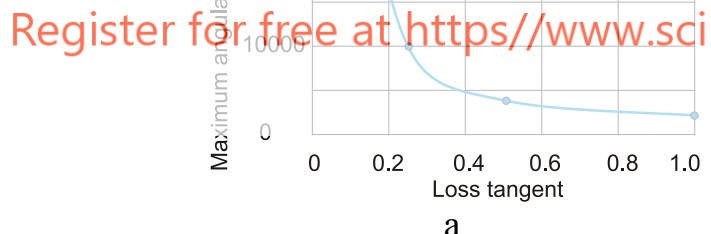

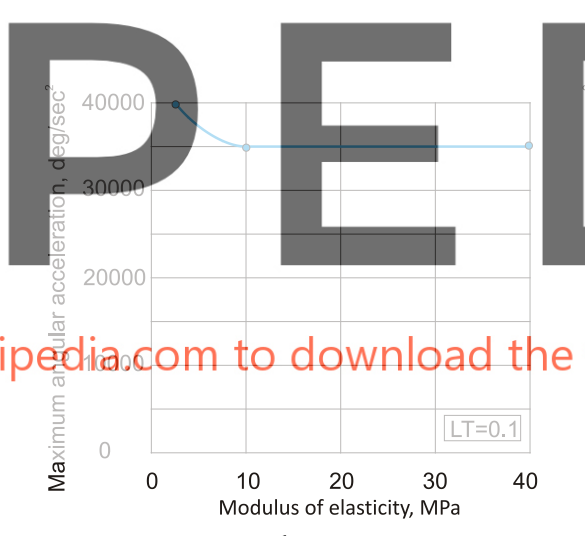

b

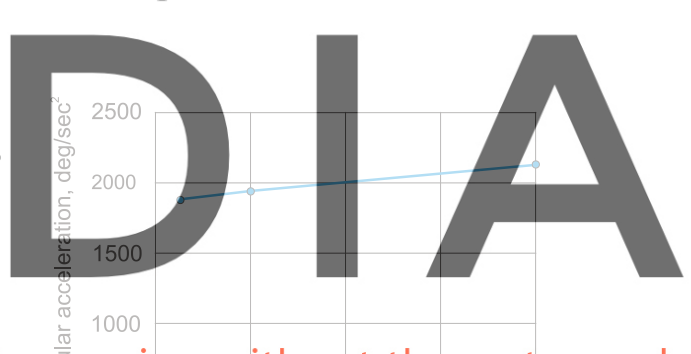

ersion without the watermark

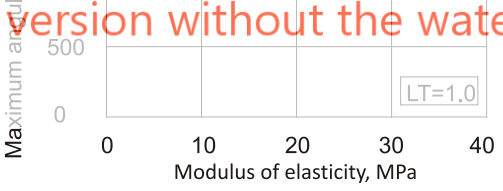

$\mathrm{C}$

Figure 5: Dependence of the maximum angular acceleration on the loss tangent (a) and the elastic modulus (b, c) of the material of the damping element

Based on the results of modeling the vibrational processes occurring in the protected module during translational vibrations of the supporting platform, we can estimate the permissible variation limit of the main mechanical characteristics of the examined device. Figure 6a shows the graphs of angular acceleration versus the position of the center of mass relative to the center of stiffness. Curves numbered 1 to 4 correspond to increasing values of the loss tangent (LT are 0.1, 0.25, 0.5 and 1 respectively). The limit value is marked with a red line. Comparison of the graphs shows that angular accelerations exceeding the limit value can occur in the protected module when the loss tangent is less than 0.5 (curves 1 and 2). Otherwise, at any position of the center of mass, the angular vibrations of the protected module will be within acceptable limits. The same conclusion can be drawn when observing 
changes in the magnitude of the angular accelerations with the variation of the damper stiffness (Figure 6b). Using a material with LT more than 0.5 ensures that the angular acceleration of the protected unit does not exceed the permissible limit (curves 3 and 4). If the loss tangent is 0.1 , the maximum allowable displacement of the center of mass is $2.6 \mathrm{~mm}$, and the maximum deviation of the damper properties is $15 \%$. The loss tangent of 0.25 corresponds to the permissible changes in these characteristics, which are $12.8 \mathrm{~mm}$ and $48 \%$, respectively.

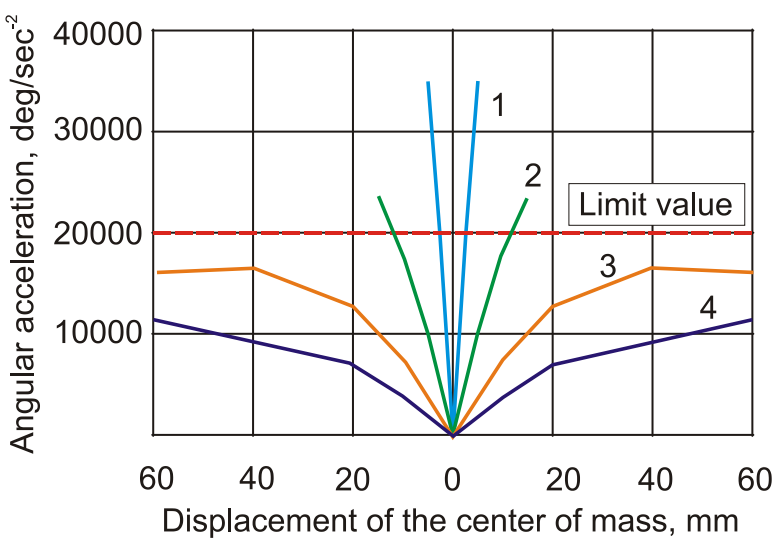

a

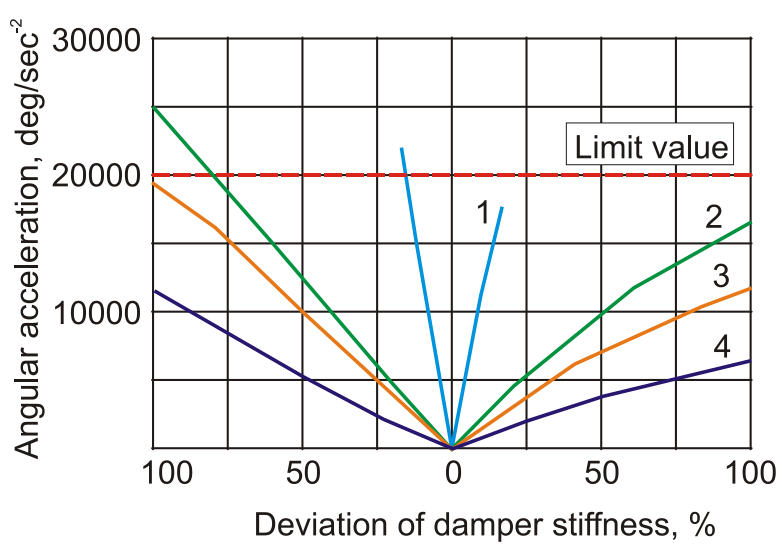

b

Figure 6: Variation in the angular acceleration of the protected unit with a change in the position of the centers of mass (a) and stiffness of the dampers (b) at different values of the loss tangent LT: 0 (curve 1), $0.25(2), 0.5(3), 1$ (4)

\section{CONCLUSIONS}

Numerical simulation of the motions of the protected solid block caused by the translational vibrations of the supporting platform revealed two significant factors affecting the value of the block angular accelerations. They are the displacement of the center of mass relative to the point of intersection of the damper axes and stiffness and the dissipative characteristics of the dampers. The permissible deviations of these characteristics from the values corresponding to the basic design have been determined. Within this range the angular acceleration of the protected module does not exceed the limiting value of $20000 \mathrm{deg} / \mathrm{sec}^{2}$ : The displacement of the center of mass should not be higher than $2.6 \mathrm{~mm}$ and the change in the stiffness of each damper should not exceed $15 \%$.

The numerical experiment has shown that the most significant effect on the angular acceleration of the protected module is produced by changes in the stiffness and dissipation coefficients of the dampers in the radial direction.

It has been found that the minimum vibration amplitudes of the protected solid block are achieved when either translational or rotation modes dominate in the natural vibration modes of the block. In this case, the corresponding natural frequencies are significantly different. When the resonant frequencies converge, the energy of the translational motion of the supporting structure is converted into the energy of rotational vibrations and the angular acceleration of the protected block increases sharply.

It has been established that an effective constructive approach, which can significantly reduce the rotational vibrations of the protected unit is an increase in the loss tangent of the 
damping element. At constant loss tangent, the change in the modulus of elasticity of the damping material does not significantly affect the value of the maximum angular acceleration.

The developed mathematical model, based on the theory of rigid body dynamics, makes it possible to analyze complex vibrations of a body installed through a suspension system on a moving base. The model, supplemented by a set of experimental data on the stiffness and dissipative properties of dampers, makes it possible to select rational parameters that ensure the permissible values of the dynamic characteristics of the system.

\section{REFERENCES}

[1] Crocker, M.J. Handbook of Noise and Vibration Control. Hoboken: Wiley, (2007).

[2] Lee, J. and Okwudire, C.E., Reduction of vibrations of passively-isolated ultra-precision manufacturing machines using mode coupling. Precis. Eng. (2016) 43: 164-177.

[3] Zhang, Y. and Gao, Z. Fiber optic gyroscope vibration error due to fiber tail length asymmetry based on elasticoptic effect. Opt. Eng. (2012) 51: 124403.

[4] Kurbatov, A.M. and Kurbatov, R.A., The vibration error of the fiber-optic gyroscope rotation rate and methods of its suppression. J. Commun. Technol. Electron. (2013) 58: 840-846.

[5] Ganiev, R.F. and Kononenko, V.O. Kolebaniya tverdykh tel (Oscillations of Solids), Moscow: Nauka (1976).

[6] Shardakov I., Glot I., Shestakov A., Gubskiy D. Vibration protection of devices sensitive to angular vibrations AIP Conference Proceedings (2020) 2312: 030004 